\title{
Influence of dislocation content on the quantitative determination of the doping level distribution in $\mathbf{n}-\mathrm{GaAs}$ using absorption mapping
}

\author{
U. Künecke ${ }^{\mathrm{a}}$ and P.J. Wellmann \\ Institute for Materials Science 6, University of Erlangen, Martensstr. 7, 91058 Erlangen, Germany
}

\begin{abstract}
Received: 16 September 2005 / Received in final form: 7 December 2005 / Accepted: 12 December 2005 Published online: 24 June 2006 - (C) EDP Sciences

Abstract. In an earlier paper [P.J. Wellmann, A. Albrecht, U. Künecke, B. Birkmann, G. Mueller, M. Jurisch, Eur. Phys. J. Appl. Phys. 27, 357 (2004)] an optical method based on whole wafer absorption measurements was presented to determine the charge carrier concentration and its lateral distribution in $n$-type ( $\mathrm{Si} / \mathrm{Te}$ ) doped GaAs. The submitted results for Si-doped GaAs gave rise to questions concerning the interpretation of absorption mappings in wafers with high dislocation densities. GaAs substrates for optoelectronic devices are strongly affected by dislocations. Therefore further studies were conducted: absorption and Hall measurements were performed on GaAs:Si wafers with high and low dislocation densities. Absorption in Si-doped GaAs is far more complex than in Te-doped GaAs. It shows a co-dependency on charge carrier concentration and dislocation content which causes complications in the quantitative optical determination of the charge carrier concentration. Qualitatively, absorption mappings depict dislocations and variations of charge carrier concentration very well.
\end{abstract}

PACS. 71.55.Eq III-V semiconductors - 78.20.-e Optical properties of bulk materials and thin films 78.40.Fy Semiconductors

\section{Introduction}

III-V compound semiconductors like GaAs and InP are of particular interest for red and near infrared light emitting device applications. Light emitting and laser diodes are usually fabricated on $n$-type doped substrates. The level of the charge carrier concentration must be known and its homogeneity across a wafer must be ensured due to their impact on the electrical properties of the devices. In order to address this issue, electrical and optical techniques have been presented, the latter having the advantages of being fast and non destructive.

In an earlier paper [1] we presented an optical technique based on absorption measurements, which aims at determining the charge carrier concentration in $n$-type GaAs:Si and GaAs:Te quantitatively. The method is based on an approach which was developed for $\mathrm{SiC}$ earlier [2,3].

The results for GaAs:Si published in [1] gave rise to questions concerning the absorption behavior of GaAs:Si in areas with high dislocation densities. Dislocation rich areas showed lower absorption at $918 \mathrm{~nm}$ than surrounding areas. Calibration data based on homogeneous samples show a general increase of absorption with increasing charge carrier concentration. The implementation of this dependency on inhomogeneous wafers would depict dislocation rich areas as areas with reduced charge carrier con-

\footnotetext{
a e-mail: ulrike.kuenecke@ww.uni-erlangen.de
}

centration. This is in contradiction to the current model of electrical active Si doping close to dislocations and PL and Raman measurements.

Therefore further studies were conducted: the approach of this work was to locate areas with high and low dislocation content via absorption mapping and conduct Hall measurements and spectrally resolved absorption spectroscopy there. This approach yields information on how absorption behavior is influenced by the presence of dislocations.

\section{Experimental setup}

All experiments were conducted on Si doped GaAs samples grown by Freiberger Compound Materials GmbH or in house [6] by VGF. Samples were polished on both sides (grade: industrial standard). Spectrally resolved absorption measurements were carried out at room temperature in the spectral range of $870 \mathrm{~nm}$ to $1500 \mathrm{~nm}$ using a PerkinElmer UV-VIS-NIR spectrometer (model: Lambda 19). All data were corrected taking into account the wavelength dependent GaAs refractive index. The absolute error of the determined absorption coefficients was $<5 \%$. Charge carrier concentration was determined by Hall measurements at room temperature (absolute error: $<10 \%$ ). Absorption mappings were acquired by a silicon gray level 
CCD camera (sensitivity: 0.5 Lux) at $918 \mathrm{~nm}(=1.351 \mathrm{eV})$, $\Delta \lambda \approx 48 \mathrm{~nm})$; the lateral resolution of the setup was $\leq 150 \mu \mathrm{m}$. The accuracy of the Hall measurements limits the detection limit of absorption mappings to $10 \%$.

Utilizing absorption mappings, samples were selected from dislocation rich regions (usually the middle and the rim of the wafer, showing a four count symmetry) and from the dislocation poor matrix. This was done for two wafers with different average charge carrier concentrations. Hall measurements and absorption spectroscopy were carried out on all samples.

\section{Results}

Absorption mappings of Si-doped GaAs wafers can show significant inhomogenities if dislocations are present. At $918 \mathrm{~nm}$, regions with high dislocation content are depicted as darker than the surrounding material (Fig. 1a, b). Absorption mappings at $1060 \mathrm{~nm}$, however, picture dislocations as brighter than the surrounding matrix (Fig. 2).

Absorption spectroscopy shows that dislocation rich areas exhibit a significantly reduced absorption close to the energy of the band gap compared to dislocation poor areas from the same wafer. This is reversed for high wavelengths (small energies). As a result the absorption curves of dislocation rich and poor areas from the same wafer intersect, explaining the observed contrast reversion between mappings taken at $918 \mathrm{~nm}$ and mappings taken at $1060 \mathrm{~nm}$. The wavelength at which the contrast reversion happens depends on the overall charge carrier concentration. It declines with increasing charge carrier concentration. The impact of dislocation content on absorption becomes smaller with increasing average charge carrier concentration of the wafer (Fig. 3).

\section{Discussion}

The observed difficulty in the interpretation of absorption mappings of dislocation rich Si-doped GaAs is related to the amphoteric behavior of $\mathrm{Si}$ in GaAs and its dependency on dislocation content.

At low doping concentrations Si substitutionally occupies Ga sites in the GaAs lattice, with progressive Si-incorporation $\mathrm{Si}$ begins to occupy As sites as well. This amphoteric behavior results in a self compensation in which free carriers provided by the $\mathrm{Si}$ donor atoms become trapped by $\mathrm{Si}$ atoms acting as acceptors. At higher Si incorporation levels neutral Si clusters and $\mathrm{Si}_{\mathrm{Ga}}$-Ga-vacancy complexes consecutively lead to further compensation [4-9]. Theoretical calculations confirm the experimental results [10]. Typical doping levels where Si acceptors, $\mathrm{Si}$ clusters and $\mathrm{Si}_{\mathrm{Ga}}-\mathrm{Ga}$-vacancy complexes are formed are given in [4]: $\mathrm{Si}_{\mathrm{As}}$ and $\mathrm{Si}$ clusters are present at an incorporated Si concentration of $2.7 \times 10^{18} \mathrm{~cm}^{-3}$ (charge carrier concentration $1.2 \times 10^{18} \mathrm{~cm}^{-3}$ ) while no $\mathrm{Si}_{\mathrm{Ga}}$-Ga-vacancy complexes can be detected. At an incorporated Si concentration of $1.3 \times 10^{19} \mathrm{~cm}^{-3}$ (charge

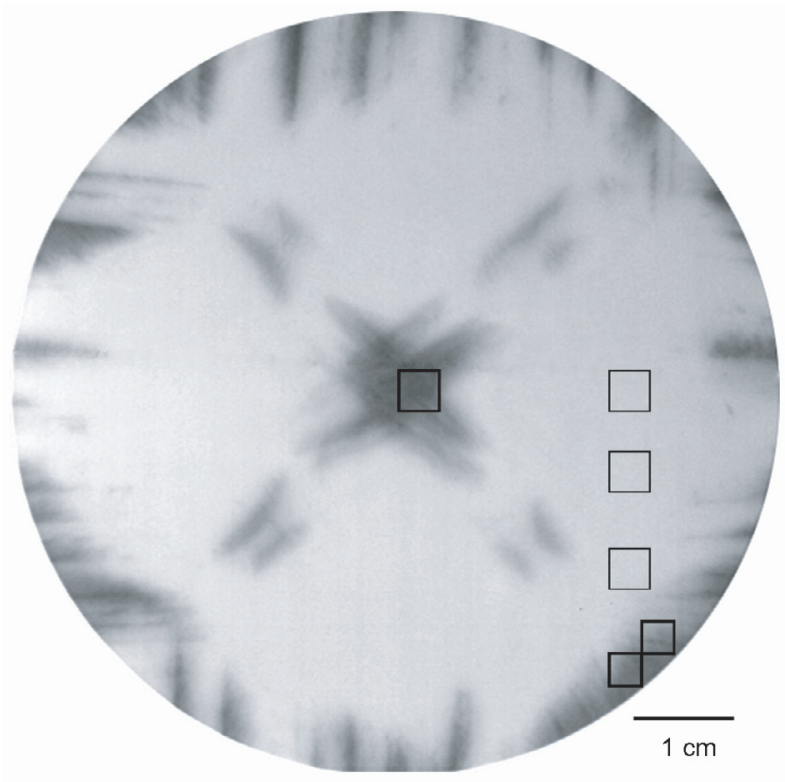

(a)

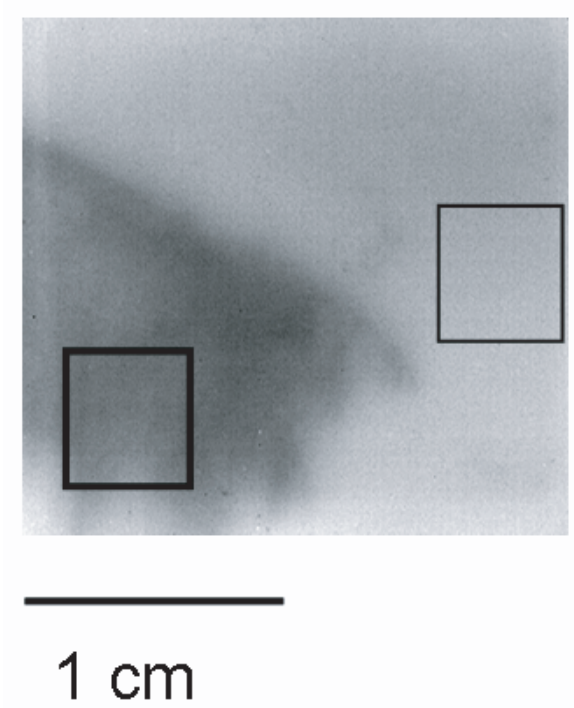

(b)

Fig. 1. (a) Absorption mapping of GaAs:Si wafer at $918 \mathrm{~nm}$, average charge carrier concentration approx. $9 \times 10^{17} \mathrm{~cm}^{-3}$, samples taken from dislocation rich areas of the wafer (bold outline) and from the matrix. (b) Absorption mapping of part of GaAs:Si wafer at $918 \mathrm{~nm}$, average charge carrier concentration approx. $3 \times 10^{18} \mathrm{~cm}^{-3}$, samples taken from dislocation rich areas of the wafer (bold outline) and from the matrix.

carrier concentration $3.7 \times 10^{18} \mathrm{~cm}^{-3}$ ) Si cluster and $\mathrm{Si}_{\mathrm{As}}$ content has further increased and $\mathrm{Si}_{\mathrm{Ga}}$-Ga-vacancy complexes are now present. At an incorporated Si concentration of $2.5-6 \times 10^{19} \mathrm{~cm}^{-3}$ (charge carrier concentration $1.2 \times 10^{18} \mathrm{~cm}^{-3}$ ) Si cluster and $\mathrm{Si}_{\mathrm{Ga}}$-Ga-vacancy content is in the range of $10^{19} \mathrm{~cm}^{-3}$, outweighing $\mathrm{Si}_{\mathrm{As}}$ by the factor 10. Birkmann et al. [6] confirm the formation of $\mathrm{Si}_{\mathrm{Ga}}$-Ga-vacancy complexes for $\mathrm{Si}$ contents between $1 \times 10^{18}$ and $1 \times 10^{19} \mathrm{~cm}^{-3}$. 
$918 \mathrm{~nm}:$

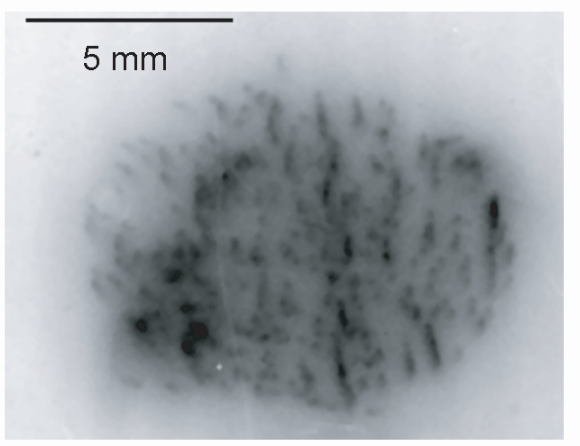

$1060 \mathrm{~nm}:$

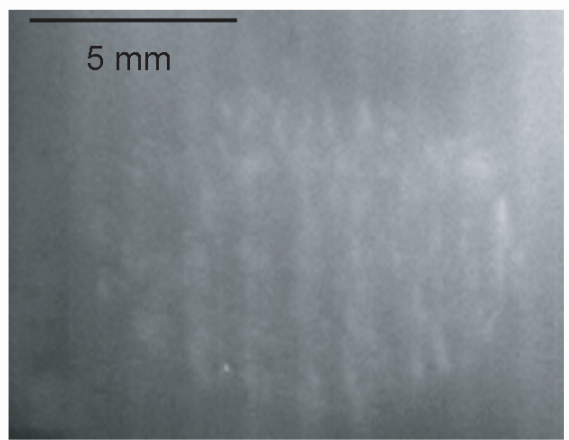

Fig. 2. Contrast reversion of absorption mapping of dislocation rich areas between $918 \mathrm{~nm}$ and $1060 \mathrm{~nm}$, GaAs:Si wafer, average charge carrier concentration approx. $8 \times 10^{17} \mathrm{~cm}^{-3}$.

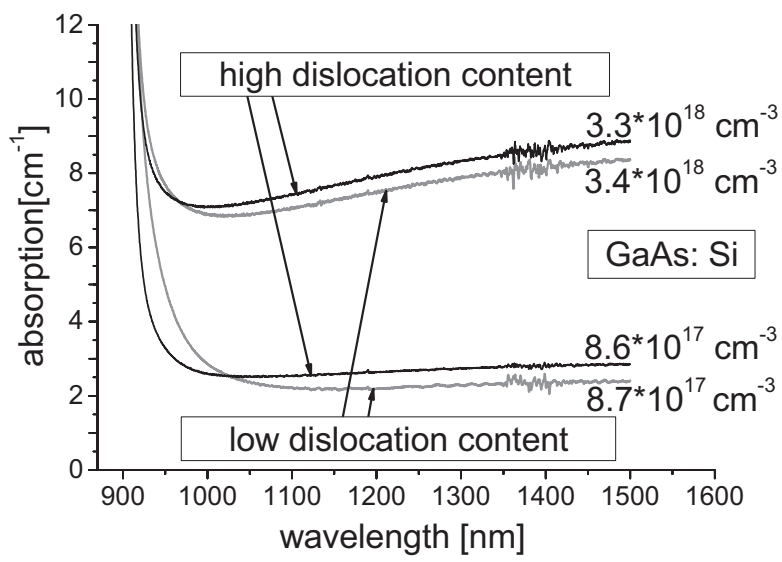

Fig. 3. Co-dependency of absorption behavior on charge carrier concentration and on dislocation density.

By contrast Te is incorporated on As sites only, Te donors are exclusively compensated by the formation of Ga vacancy-donor complexes [11].

Dislocation content influences the degree of self compensation of Si in GaAs. Defect atmospheres surrounding dislocations in $\mathrm{Si}$ and Te doped GaAs have been investigated by various methods like EBIC [12], DSL photo etching $[12,14]$, PL $[12,15]$, phase contrast microscopy [15] and Micro-Raman analysis [13-15]. The various results show that in the case of $\mathrm{Si}$ doping the defect atmosphere is characterized by an increase, in the case of Te doping by a decrease of the charge carrier concentration compared to the surrounding material. This is caused by a depletion of dopants in the As-sublattice. Since Si acts as an acceptor on As sites, depletion will result in a reduced self compensation around dislocations causing an increase of the charge carrier concentration. The typical extension of the denuded dislocation atmosphere of more than $10 \mu \mathrm{m}$ is explained by a fast interstitial type diffusion. Interstitials move to the dislocation and replace the dopant atom on a substitutional site in the As-sublattice. The dopant now occupying the interstitial position becomes a fast diffuser [13].
Absorption will depend on the band structure and manifest itself as a result of either (i) valence to conduction band transitions (fundamental absorption), (ii) transitions between acceptor levels and conduction band and transitions between valence band and donor levels (below band gap absorption) and (iii) free carrier absorption resulting from intra valence band transitions (as discussed for $\mathrm{SiC}$ in $[2,3])$. Concerning its fundamental absorption (i) GaAs shows a doping level induced band gap shift. Due to effects like doping induced band gap shrinkage and band tailing an initial decrease of the band gap is observed with increasing doping level. With increasing charge carrier concentration band filling sets in and interferes with the former, finally resulting in an increase of the optical band gap. For an overview see Hill [16] and Casey [17]. Because of this the wavelength region close to the band gap cannot be used for the optical determination of the charge carrier concentration.

Concerning below band gap absorption (ii), the absorption will depend on the presence of donor and acceptor levels. Because $\mathrm{Si}$ is incorporated as donor and acceptor, below band gap absorption in GaAs:Si will be higher compared to GaAs doped with the non-amphoteric donor Te. Since the self compensation of Si is reduced along dislocations, absorption in GaAs:Si will be influenced by the presence of dislocations.

It is clearly visible from Figure 3 that areas with high and low dislocation densities from the same wafer show a significantly different absorption behavior as a function of the wavelength of the incoming light: high dislocation content results in reduced near band gap absorption caused by the depletion of $\mathrm{Si}$ acceptors in the denuded zones around dislocations. High dislocation content results in an increased absorption of free charge carriers since self compensation is reduced. Small dislocation content results in increased near band gap absorption because of the presence of acceptor levels and in decreased absorption of free charge carriers because of the higher degree of self compensation. The respective absorption curves intersect, contrast reversion of dislocations in absorption mappings is the result (Fig. 2). 


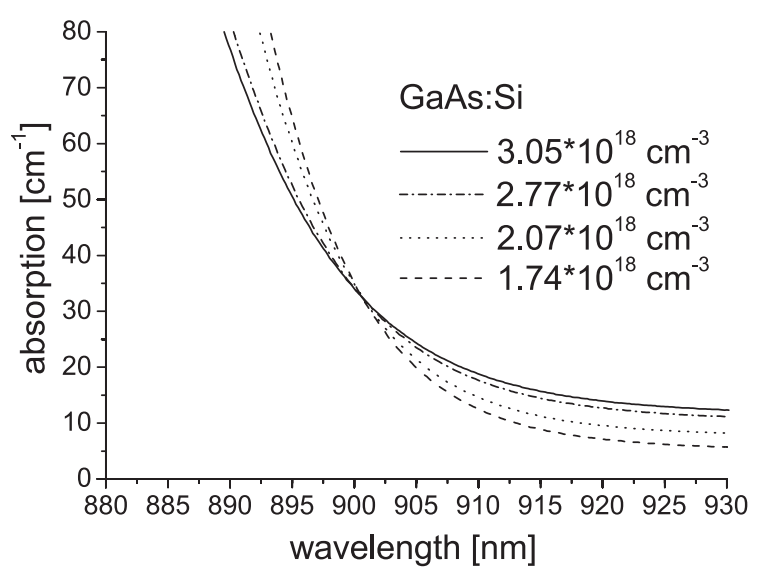

(a)

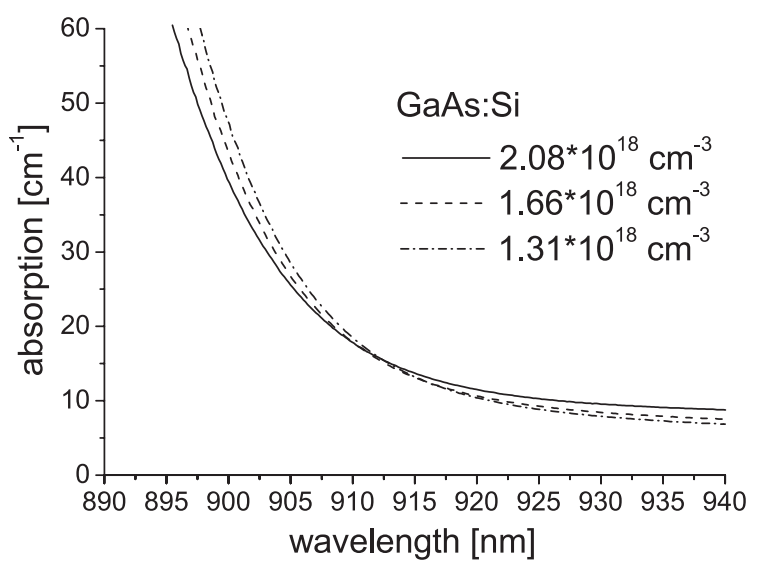

(b)

Fig. 4. (a) High dislocation content, effect of band-filling on absorption as a function of charge carrier concentration. (b) Low dislocation content, effect of band-filling on absorption as a function of charge carrier concentration.

The wavelength, at which this contrast reversion happens, depends not only on the dislocation content but also on charge carrier concentration. The reason is that with increasing charge carrier concentration the above mentioned band-filling will prevail over the initial band gap shrinkage and change the shape of the absorption curve. We observe this for charge carrier concentrations bigger than approximately $n=1.4 \times 10^{18} \mathrm{~cm}^{-3}$ [1] for dislocation rich and poor samples (Fig. 4). With further increasing charge carrier concentration the absorption edges shift towards smaller wavelengths, the inclination of the respective absorption curves drops. This causes the intersection of absorption curves belonging to differently doped samples.

Intersection of absorption curves is a general problem for the optical determination of charge carrier concentration because the correlation between charge carrier concentration and absorption coefficient needs to be unique for the chosen wavelength for a wide range of charge carrier concentrations and dislocation contents.

\section{Resume: mapping of Si doped versus Te doped GaAs wafers}

While the quantitative optical determination of the charge carrier concentration via absorption mapping can be applied for Te doped GaAs, the method cannot be used as easily for GaAs:Si. Reason is the much more complex compensation mechanism of $\mathrm{Si}$ in GaAs compared to Te in GaAs. Different degrees of compensation resulting from variations in dislocation content cause different principal shapes of the respective absorption curves. The unique correlation between absorption coefficient and charge carrier concentration cannot be established for a wide range of charge carrier concentrations, unknown degree of compensation and unknown dislocation content at wavelengths in the range of a silicon CCD camera. With the existing sample material it cannot be assured yet, if a mapping taken at a single wavelength well away from the point of intersection of the absorption curves (e.g. $1500 \mathrm{~nm}$ ) can exclude the influence of different dislocation content and allow the single wavelength quantitative determination of charge carrier concentration. The formation of Si clusters in very highly compensated material might pose a further complication. The presence of Si clusters would increase the absorption coefficient at constant or even declining charge carrier concentrations.

The dependency of the absorption coefficient on the wavelength of the incoming light as measured in spectroscopy is very significant: from the shape of the curve one can clearly conclude on the presence of dislocations, from its relative height on the amount of charge carrier concentration. A quantitative analysis of both would entail the necessity to analyze the height of the absorption coefficient at a given wavelength (e.g. $1000 \mathrm{~nm}$ ) and the incline of the curve at this point.

While the quantitative optical determination of charge carrier concentration is difficult for Si doped GaAs, qualitatively, a single wavelength (e.g. $918 \mathrm{~nm}$ ) absorption mapping provides valuable information on the presence and exact location of dislocations and on the homogeneity of charge carrier concentration in a fast and easy way. It can facilitate a pass-no pass decision in quality control. The dependency of absorption on dislocation content implies the possibility to determine the dislocation content via spectrally resolved absorption measurements.

The authors would like to thank U. Kretzer and M. Jurisch (Freiberger Compound Materials GmbH) and B. Birkmann and G. Müller (Institute for Materials Science 6) for GaAs samples.

\section{References}

1. P.J. Wellmann, A. Albrecht, U. Künecke, B. Birkmann, G. Mueller, M. Jurisch, Eur. Phys. J. Appl. Phys. 27, 357 (2004)

2. R. Weingärtner, P.J. Wellmann, M. Bickermann, D. Hofmann, T.L. Straubinger, A. Winnacker, Appl. Phys. Lett. 80, 70 (2002) 
3. P.J. Wellmann, R. Weingärtner, Mat. Sci. Eng. B 102, 262 (2003)

4. C. Domke, Ph. Ebert, M. Heinrich, K. Urban, Phys. Rev. B 54, 10288 (1996)

5. C. Domke, Ph. Ebert, K. Urban, Phys. Rev. B 57, 4482 (1998)

6. B. Birkmann, R. Weingärtner, P. Wellmann, B. Wiedemann, G. Müller, J. Cryst. Growth 237-239, $345(2002)$

7. M. Baeumler, M. Maier, N. Herres, Th. Bünger, J. Stenzenberger, W. Jantz, Mat. Sci. Eng. B 91-92, 16 (2002)

8. S. Schuppler, D.L. Adler, L.N. Pfeiffer, K.W. West, E.E. Chaban, P.H. Citrin, Phys. Rev. B 51, 10527 (1995)

9. J. Gebauer, R. Krause-Rehberg, C. Domke, Ph. Ebert, K. Urban, Phys. Rev. Lett. 78, 3334 (1997)
10. J.E. Northrup, S.B. Zhang, Phys. Rev. B 47, 6791 (1993)

11. J. Gebauer, E.R. Weber, Appl. Phys. Lett. 82, 2059 (2003)

12. J.L. Weyher, P.J. van der Wel, C. Frigerl, Semicond. Sci. Tech. 7, A294 (1992)

13. O. Paetzold, G. Irmer, J. Monecke, S. Griehl, J. Raman Spectrosc. 24, 761 (1993)

14. O. Paetzold, K. Sonnenberg, G. Irmer, Mat. Sci. Eng. B 44, 217 (1997)

15. O. Paetzold, K. Sonnenberg, G. Irmer, Inst. Phys. Conf. Ser. 160, 119 (1997)

16. D.E. Hill, Phys. Rev. A 133, 866 (1964)

17. H.C. Casey Jr., D.D. Sell, K.W. Wecht, J. Appl. Phys. 46, $250(1975)$ 